\title{
An alternative formulation of Lyapunov exponents for computing Lagrangian coherent structures
}

\author{
Allen R. Sanderson* \\ Scientific Computing and Imaging Institute, University of Utah, USA
}

\begin{abstract}
Lagrangian coherent structures are time-evolving surfaces that highlight areas in flow fields where neighboring advected particles diverge or converge. The detection and understanding of such structures is an important part of many applications such as in oceanography where there is a need to predict the dispersion of oil and other materials in the ocean. One of the most widely used tools for revealing Lagrangian coherent structures has been to calculate the finite-time Lyapunov exponents, whose maximal values appear as ridgelines to reveal Lagrangian coherent structures. In this paper we explore an alternative formulation of Lyapunov exponents for computing Lagrangian coherent structures.
\end{abstract}

\section{INTRODUCTION}

Understanding transport and mixing in flow fields is a fundamental task in many computational fluid dynamics (CFD) applications. For instance, oceanographers wish to better understand tidal inlets and their circulation patterns. One such feature of interest in this pursuit is Lagrangian coherent structures (LCS) [5]. In time-varying systems, LCS are the boundaries between dynamically different regions. These structures can be defined in terms of finite-time Lyapunov exponents (FTLE) [9], whose maximal values appear as ridgelines to reveal LCS. One of the benefits of calculating FTLE is that they reveal geometry that can not be easily seen in the flow field or via the trajectories that lie within the field, see for example the work of Garth among others [3, 4].

Lyapunov exponents (LE) quantify the rate of separation of infinitesimally close trajectories over infinite time and are a measure of the chaos in the system. While this quantity can reveal structures not otherwise seen in the flow field understanding their origin can be difficult because of the Lagrangian nature of the calculation. In this short note we propose an alternative formulation that while it has a Lagrangian basis can also be viewed using an Eulerian basis. Having this duality we believe provides complementary information in understanding the location of LCS. There are other formations of Lyapunov exponents such as the finite-size Lyapunov exponents (FSLE) [1] and have been compared to finite-time Lyapunov exponents (FTLE) [6]. Others have explored exponents based on streaklines (as oposed to stream or path lines) [7] or used variance analysis [8].

\section{Calculating lCS via fTLE}

For background we give a brief overview of calculating FTLE. For more in-depth background the reader is referred to [5,9]. For a smooth vector field of the form:

$$
\dot{x}=v(x, t), x \in V \subset \Re^{n}
$$

At a time $t$ the path of a particle is defined to be $x\left(t, t_{0}, x_{0}\right)$ initialized at $x_{0}$ and time $t_{0}$. We define the flow map to map the initial particle location $x_{0}$ to its location at time $t$ :

*e-mail: allen@sci.utah.edu

$$
F_{t_{0}}^{t}: U \mapsto U
$$

The flow map encodes the Lagrangian particle path, is continuously differentiable, and can be deformed. The deformation gradient $\nabla F_{t_{0}}^{t}$ is defined to be the right Cauchy-Green strain tensor:

$$
C_{t_{0}}^{t}=\left[\nabla F_{t_{0}}^{t}\right]^{T} \nabla F_{t_{0}}^{t}
$$

The FTLE associated with a particle is defined to be proportional to the largest eigen value of this tensor:

$$
\Lambda_{t_{0}}^{t}=\frac{1}{t} \log \sqrt{\lambda_{\max }\left[C_{t_{0}}^{t}\right]}
$$

The LCS are the locally maximizing surfaces or ridges in the scalar field $\Lambda_{t_{0}}^{t}$. Repelling LCS are found by integrating forward in time while attracting LCS can be found by integrating backward in time.

In Figure 1 we show an example of an instaneous FTLE visualization for a river inlet during slack tide. The image clearly shows a strong coherent structure as a red ridge line, and is the instanteous slack tide interface between the river and the ocean as well as other weaker structures in yellow and green.

\section{Calculating lcs via a Euclidean distance mea- SUREMENT}

In oceanography, drifters are often used to understand the dynamics of ocean-atmosphere interaction [2]. Drifters are placed in arrays in the ocean and then tracked over time. Ocean currents can then be determined by the GPS paths recorded from the drifters. One of the key measurements is the distance traveled by the drifter from its initial location. We use a similar distance measurement in our formulation for finding LCS. That is we measure the distance between the seed point and the end point of the streamline.

This distance measure is similar to the distance used in the FTLE calculation, where one looks at the inter-particle divergence. However, instead of the inter-particle divergence we are looking at the intra-particle divergence. That is the overall Euclidean distance the particle has traveled.

Physically the distance traveled, when small can be an indication of a particle being trapped in a eddy. However, it can also simply mean a particle is moving very slowly from its initial location.

We define the distance map to map the Euclidean distance traveled by a particle from its initial location $x_{0}$ at time $t_{0}$ to its location at time $t$ :

$$
L_{t_{0}}^{t}: V \mapsto V
$$

The distance map unlike the flow map does not encode the Lagrangian particle path. However, it is continuously differentiable and can be deformed. The deformation gradient $\nabla L_{t_{0}}^{t}$ is a vector and is similar in nature to the eigen values of the right CauchyGreen strain tensor:

$$
D_{t_{0}}^{t}=\nabla L_{t_{0}}^{t}
$$

The Lyapunov exponent associated with a particle is defined to be proportional to the largest gradient value: 


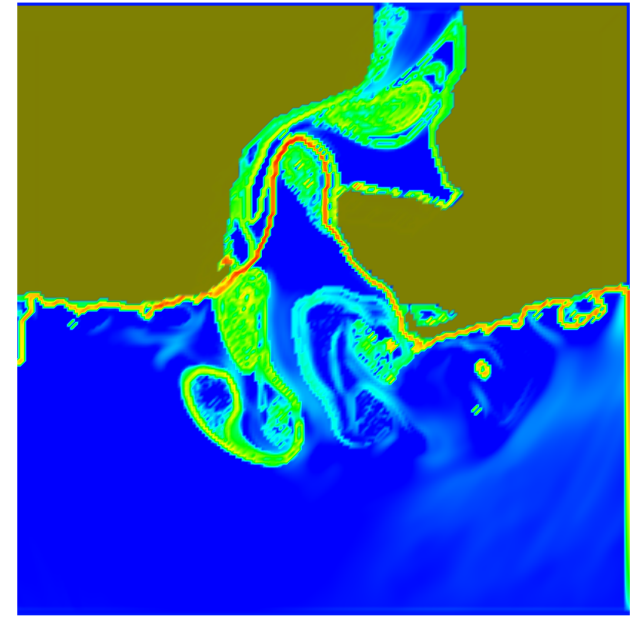

Figure 1: A traditional FTLE image of an instaneous 3D flow field for the New River Inlet, Onslow, North Carolina. The dominant ridgeline demarcates the slack tide interface between the river and the ocean.

$$
\Gamma_{t_{0}}^{t}=\frac{1}{t} \log \sqrt{\gamma_{\max }\left[D_{t_{0}}^{t}\right]}
$$

Like a traditional FTLE we also limit the advection based on the integration time. While other limits are possible using the integration time allows a one-to-one comparison of the Euclidean distance measure to FTLE. Thus the only difference in the systems is the maximal value calculation.

In Figure 2 a visualization using an Euclidean distance measure for the same river inlet as in Figure 1 is shown. The image clearly shows the same the strong coherent structure as a red ridge line, the slack tide interface between the river, as well as other weaker structures in yellow and green. We will discuss further similarities and differences in Section 6

\section{GaINING INCITE INTO LCS}

In a typical analysis exploration before a scientist creates a FTLE image they often start by visualizing a series of streamlines (in the instantaneous case) colored by their arc length, Figure 3. Though such visualizations can show flow features such as eddies they can be difficult to fully interpret due to flow field complexities.

At the same time it is possible to create a scalar image with the seed points colored by the arc length of the streamlines that would be used in the FTLE calculation, Figure 4. Such an image gives an Eulerian view of the system and can give incite into the origin of where particles travel the longest using an arc length measure within a given time period. While such a scalar image gives incite into the speed it is difficult to understand the origin of the coherent structures such as those found in Figure 1.

In Figure 5 the same streamlines are visualization as in Figure 3 except the color map is based on the Euclidean distance traveled by a particle. The first obvious difference is that the range of values is more evenly distributed compared to the arc lengths that are dominated by a few values. The second difference are the coherent regions where particles travel the least and furthest from their initial location. In the case of the latter one can clearly see the main channel. For the former, there are two eddies just outside of the river inlet where particles remain for a long time traveling very little from their initial starting locations. Only one of the eddies has similar structure when viewed using an arc length measure.

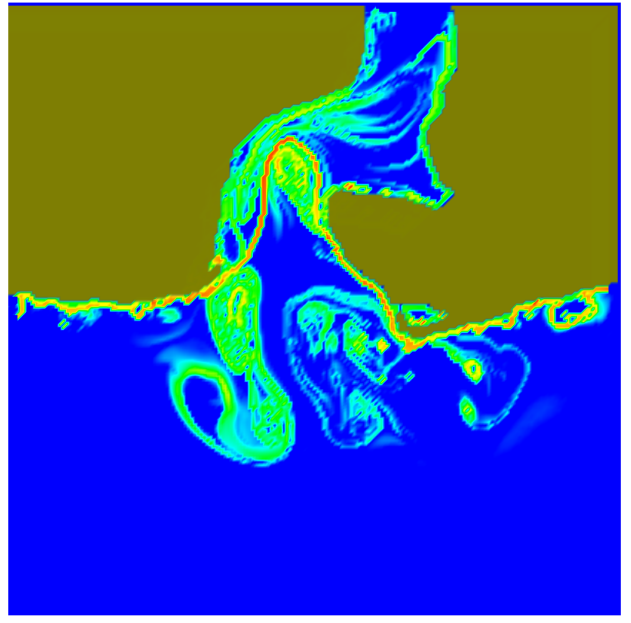

Figure 2: A FTLE like image of 3D flow field for the New River Inlet, Onslow, North Carolina using a Euclidean distance measure. The dominant ridgeline demarcates the slack tide interface between the river and the ocean.

In Figure 6 the seed points are colored by the Euclidean distance traveled by the particle originating at that location and gives an $\mathrm{Eu}$ lerian view of the system. These distances are also used for the Lyapunov exponent calculations. This image differs greatly from the one based on the arc length shown in Figure 4. For instance, the location of the slack tide river-ocean interface is clearly seen and is where the coherent structure is located as seen in Figure 2.

\section{APPLICATION tO TIME VARYING FIELDS}

We now apply our Euclidean distance measure to time varying fields and construct similar images as for the instantaneous example. In Figures 7 and 9 the seed points are colored based on the arc length and Euclidean distance respectively. Remarkably the images obtain are very similar with only slight differences. In Figures 8 and 10 we compute the associated Lyapunov exponents over the same time period. Again remarkably similar ridgelines are obtained.

\section{Discussion}

In Figures 1 and 2 we showed the traditional and Euclidean distance based Lyonapuv exponents images. While the images reveal similar instantaneous structures we do not believe this to always be the case. In fact, it should not be the case because the underlying basis for the exponent is an inter- and intra-distance measure and are fundamentally different. The question that remains to be answered is what does our Euclidean distance physically describe for a dynamical system? In contrast, a traditional FTLE that has its roots well established as the basis for describing the entropy and other measures in a dynamical system. As such, while promising we must understand the exponent's meaning.

At the same time the coloring of the seed locations based on the arc length and distance traveled for the instantaneous field in Figures 4 and 6 respectively, provide complementary flow field information. Though there are subtle differences in Figures 7 and 9, one can not conclude the same technique provides additional information for the time varying field. This result is perhaps not surprising and requires further investigation to fully understand.

\section{Conclusion}

We have shown an alternative formulation of Lyapunov exponents using an Euclidean distance measure. The resulting exponents form 


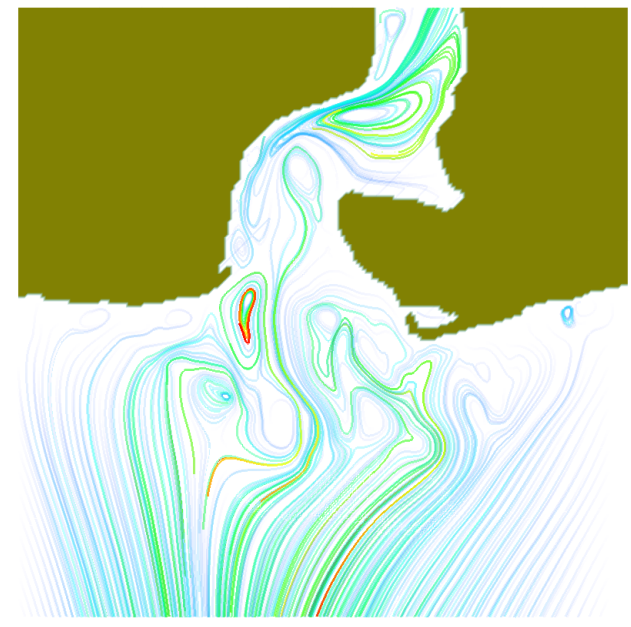

Figure 3: A series of 3D streamlines for the flow field from the New River Inlet, Onslow, North Carolina. The streamlines are colored via the arc length (blue: short distances, red: long distances) and show a series of eddies. None of which are more dominant than another.

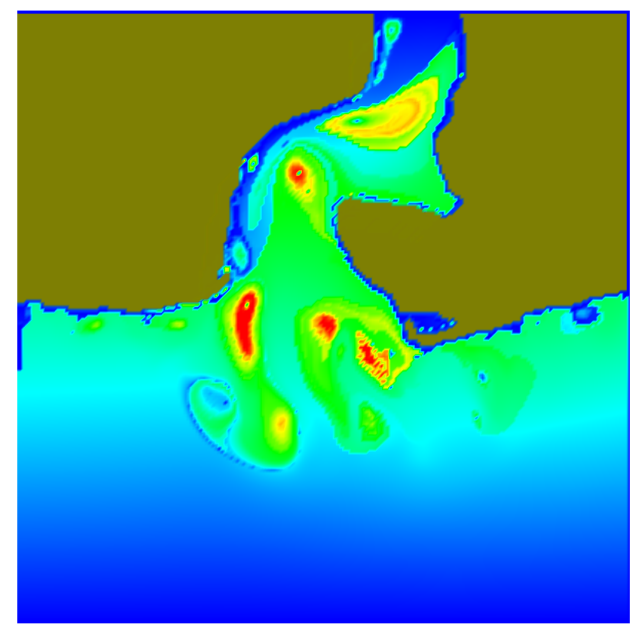

Figure 4: An image of the seed points colored by the arc length of the streamline originating at the seed. The red regions represent particles that have travel the furthest over a finite time period. Whereas the blue regions represent particles that have travel the least.

ridgelines that represent Lagrangian coherent structures like traditional FTLE images. We have demonstrated that in the instantaneous case that the underlying measurement, the Euclidean distance can reveal additional information about the location of coherent structures. Though we have compared our alternative formulation to traditional FTLE we do not see being a replacement but rather one that provides complementary information about the flow dynamics.

\section{ACKNOWLEDGEMENTS}

The author wishes to thank Tuba Ozkan-Haller and Saeed Moghimi of the Northwest National Marine Renewable Energy Center for the hydrodynamic data and Filip Sadlo for the initial discussions. This work was supported in part by a grant from the United States De-

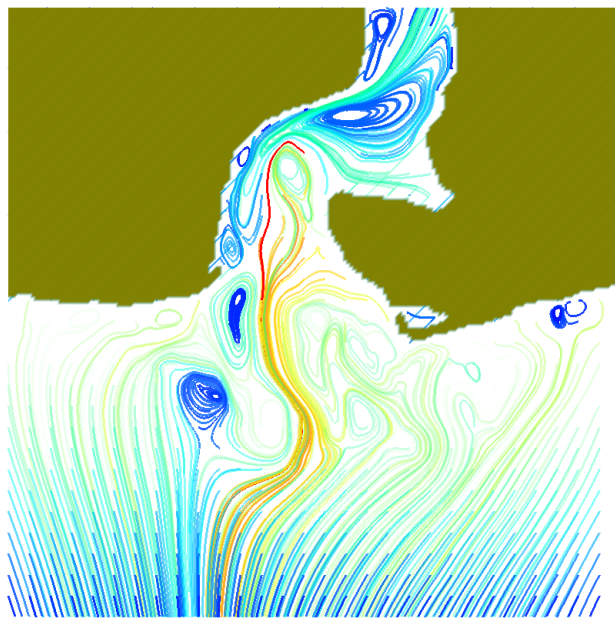

Figure 5: A series of 3D streamlines for the flow field from the New River Inlet, Onslow, North Carolina. The streamlines are colored based on the Euclidean distance a particle would travel from its initial starting location (blue: short distances, red: long distances).

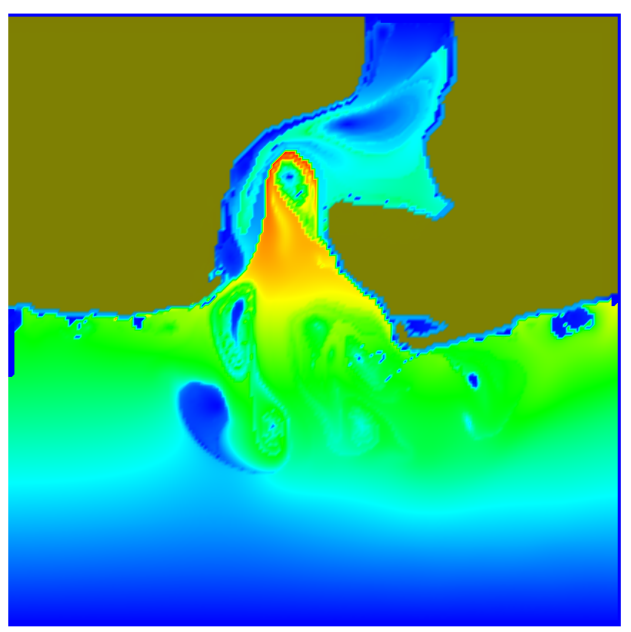

Figure 6: An image of the seed points colored by the Euclidean distance a particle would travel from the seed point using an instantaneous flow field. The red regions represent particles that have trave the furthest over a finite time period. Whereas the blue regions represent particles that have travel the least.

partment of Energy National Energy Technology Laboratory Program.

\section{REFERENCES}

[1] G. Boffetta, P. Giuliani, G. Paladin, and A. Vulpiani. An extension of the lyapunov analysis for the predictability problem. Journal of the Atmospheric Sciences, 55(23):3409-3416, 2013/11/15 1998.

[2] Y. Chang, D. Hammond, A. Haza, P. Hogan, H. Huntley, A. K. Jr., B. L. Jr., V. Taillandier, A. Griffa, and T. zgkmen. Enhanced estimation of sonobuoy trajectories by velocity reconstruction with near-surface drifters. Ocean Modelling, 36(34):179 - 197, 2011.

[3] C. Garth, F. Gerhardt, X. Tricoche, and H. Hans. Efficient computation and visualization of coherent structures in fluid flow applica- 


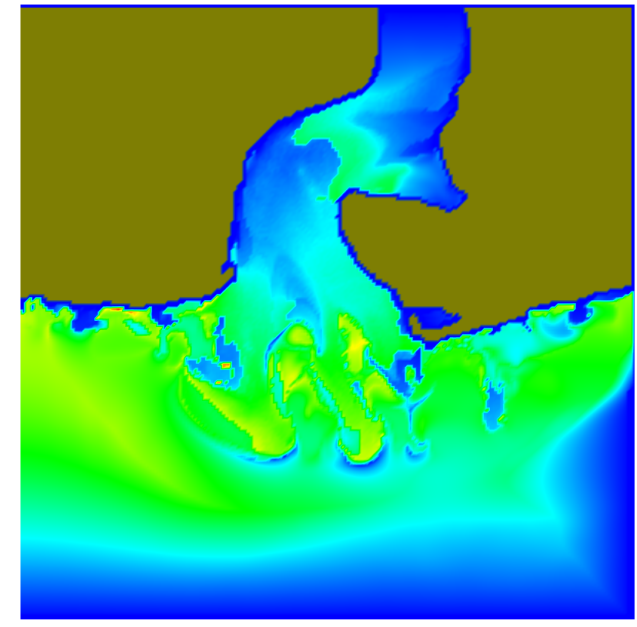

Figure 7: An image of the seed points colored by the arc length of the pathline originating at the seed. The red regions represent particles that have travel the furthest over a finite time period. Whereas the blue regions represent particles that have travel the least.

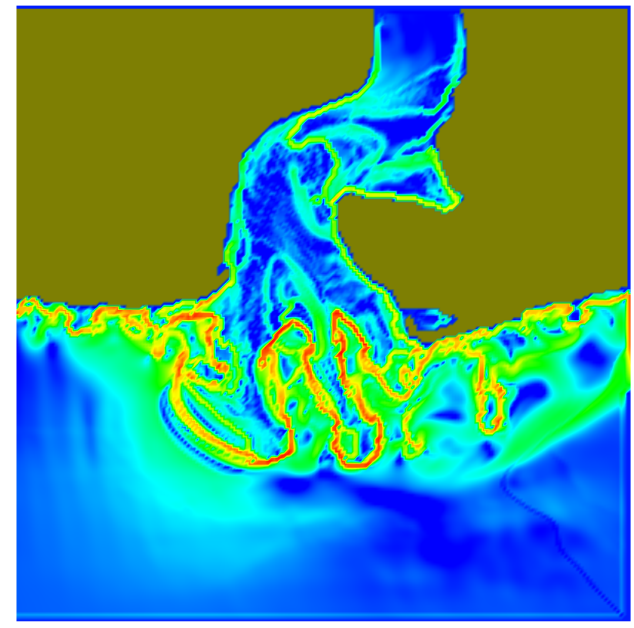

Figure 8: A traditional time varying FTLE image of 3D flow field. The dominant ridgeline demarcates the interface between the river and the ocean.

tions. IEEE Transactions on Visualization and Computer Graphics, 13(6): 1464-1471, 2007

[4] C. Garth, G.-S. Li, X. Tricoche, C. Hansen, and H. Hagen. Visualization of coherent structures in transient $2 \mathrm{~d}$ flows. In H.-C. Hege, K. Polthier, and G. Scheuermann, editors, Topology-Based Methods in Visualization II, Mathematics and Visualization, pages 1-13. Springer Berlin Heidelberg, 2009.

[5] G. Haller and G. Yuan. Lagrangian coherent structures and mixing in two-dimensional turbulence. Physica D: Nonlinear Phenomena, 147(34):352 - 370, 2000

[6] R. Peikert, A. Pobitzer, F. Sadlo, and B. Schindler. A comparison of finite-time and finite-size lyapunov exponents. In P.-T. Bremer, I. Hotz, V. Pascucci, and R. Peikert, editors, Topology-Based Methods in Visualization III, Mathematics and Visualization, pages 1-14. Springer Berlin Heidelberg, 2013.

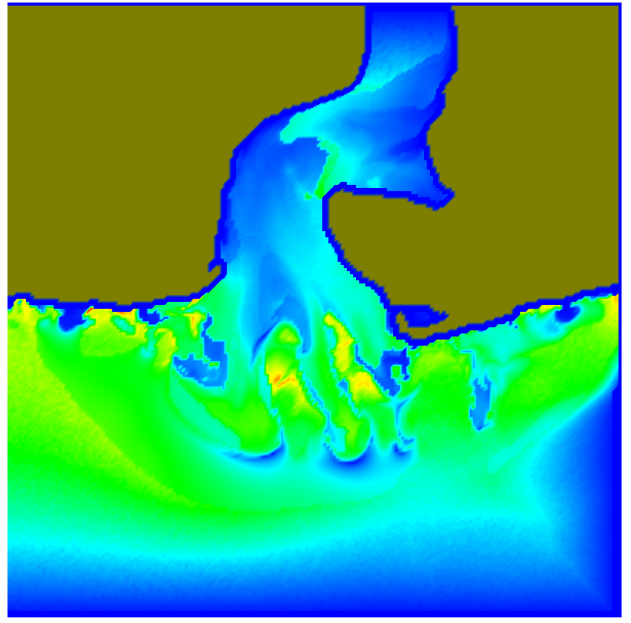

Figure 9: An image of the seed points colored by the Euclidean distance a particle would travel from the seed point using a time varying flow field. The red regions represent particles that have travel the furthest over a finite time period. Whereas the blue regions represent particles that have travel the least.

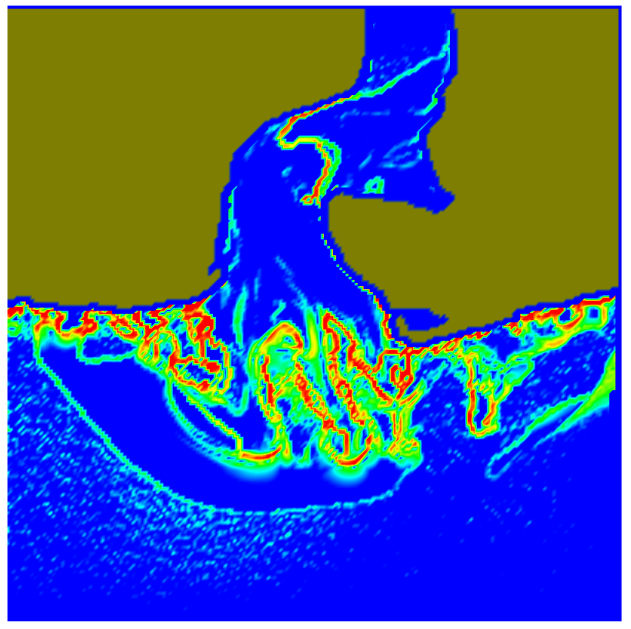

Figure 10: A time varying LE image using a Euclidean distance measure. Similar structures as well as other structures can be seen when compared to the traditional FTLE in Figure 8.

[7] F. Sadlo and D. Weiskopf. Time-dependent 2-d vector field topology: An approach inspired by lagrangian coherent structures. Computer Graphics Forum, 29(1):88-100, 2010.

[8] D. Schneider, J. Fuhrmann, W. Reich, and G. Scheuermann. A variance based ftle-like method for unsteady uncertain vector fields. In R. Peikert, H. Hauser, H. Carr, and R. Fuchs, editors, Topological Methods in Data Analysis and Visualization II, Mathematics and Visualization, pages 255-268. Springer Berlin Heidelberg, 2012.

[9] S. C. Shadden, F. Lekien, and J. E. Marsden. Definition and properties of lagrangian coherent structures from finite-time lyapunov exponents in two-dimensional aperiodicflows. Physica D: Nonlinear Phenomena, 212(34): $271-304,2005$. 